\title{
SPIRHAPALUM POLESIANUM N. G., N. SP. TRÉMATODE DU SANG D'EMYS ORBICULARIS L.
}

\author{
Par L. EJSMONT
}

\section{INTRODUCTION}

Les publications récentes des auteurs américains, concernant les nouveaux trématodes rencontrés dans le système sanguin des tortues, m'ont incité à profiter du matériel reçu occasionnellement de Polesie (Pologne) et comprenant un exemplaire de tortue. L'autopsie a eu lieu le 11 avril 1924 et la dilacération du cøeur a mis en liberté un ver. Le premier examen microscopique m'a déjà révélé dans le ver la présence du contenu intestinal noir, provenant de la décomposition des érythrocytes, ainsi. que les rangées des poils bacilliformes dont le corps du ver est orné, caractères particuliers aux trématodes du sang. L'examen de la rate y dévoila également la présence d'un ver plus petit que le précédent et qui paraissait ne pas avoir atteint sa maturité sexuelle, mais qui se montrait, quant aux autres caractères, identique au précédent en miniature. Dans le tissu de la rate, ainsi que dans celui du foie, étaient disséminés des oufs du ver, de forme ovale et couverts d'une coque transparente et très délicate. Ces œufs accusaient un commencement de développement, caractérisé par des embryons en forme de sphères cellulaires. Un second exemplaire du même parasite adulte fut trouvé dans le cour de la tortue le 17 avril et, le 26 juin 1924, un exemplaire immature fut rencontré dans la rate. Les parasites étaient accompagnés dans les trois cas d'œufs disséminés dans les tissus examinés. Mon étude s'arrêta là et fut réprise le 18 mai 1925.

J'ai examiné en tout 31 tortues, longues de $23 \mathrm{~cm}$. à $30 \mathrm{~cm}$., 5 et larges de $12 \mathrm{~cm}$. à $13 \mathrm{~cm}$., 5. Tous ces animaux provenaient de la province de Polesie. Parmi eux, six contenaient des parasites dont six adultes placés dans le cour, tandis que deux autres jeunes étaient dans la rate de deux tortues. Les huit parasites furent préparés en entier et colorés au carmin boracique ou aluné. L'examen de tous les principaux organes m'a permis de reconnaître qu'il s'agissait d'un nouveau genre. Je voudrais adresser ici ma vive reconnaissance à $M$. le prof. K. Janicki, directeur de Laboratoire de Zoologie, qui a bien voulu s'intéresser à mes recherches pendant

Andales de Parasitologie, T. V, $\mathrm{N}^{\circ} 3 .-1^{\text {er }}$ juillet 1927 , p. 220-235. 
mon séjour à ce Laboratoire ainsi qu'à M. le prof. W. Stefanski, directeur de Laboratoire de Zoologie et de Parasitologie vétérinaire.

Ce parasite appartient, comme tous les trématodes du sang des tortues, à la famille des Spirorchidæ Stunkard, 1921. Voici la traduction des caractères donnés par l'auteur pour cette famille :

"Trématodes allongés à faible musculature, munis d'une ou de deux petites ventouses et vivant dans le sang de la tortue. Absence de pharynx. Nombreux testicules lobés, dont la masse se trouve placée en avant ou parfois en arrière par rapport à l'ovaire. Ce dernier est lobé. Le canal de Laurer est présent. Utérus court. Les oufs sont grands, à coque épaisse. Ces œufs sont déposés isolément (1). "

Toutes les formes appartenant à cette famille proviennent certainement du système circulatoire, abstraction faite des conditions dans lesquelles ils furent découverts, car on les observa le plus souvent dans les poumons, comme c'est le cas pour les A porocotylidx, rencontrés souvent dans les branchies (Odhner, 1900 ; Ejsmont, 1925) (2).

Jusqu'à présent, les formes suivantes ont été décrites :

Famille Spirorchidx Stunkard, 1921 (3).

Sous-famille Spirorchine Stunkard, 1921.

Genre Spirorchis Mac Callum, 1918.

Espèces : S. innominala Ward, $1921(=S$. euslreplos Mac Callum, 1921).

S. artericola (Ward, 1921).

S. emydis Mc Cal., 1921.

S. scripla Stunk., 1923.

S. elegans Stunk., 1923.

S. picla Stunk., 1923.

S. pictr Mac Cal., 1926.

S. chelydræ Mac Cal., 1926.

S. blandingi Mac Cal., 1926.

Genre Henolosoma Stunk., 1922.

Espèce: H. hæmatobium Stunk., 1922.

Genre Hrmalolrema Stunk., 1923.

Espèce: H. parvum Stunk., 1923.

(1) Les œufs du ver faisant l'objet de cette étude possèdent une coque très fine. D'ailleurs, Stunkard (1926) lui-même fait observer que la diagnose de la famille doit être changée, parce que la nouvelle forme Unicœeum ruszkowskii Stunk. se distingue par le manque du canal de Laurer, par l'ovaire différent et par les cufs à coque tine.

(2) Odhner (1912) est du reste du même avis en ce qui concerne les Schistosomida trouvés parfois dans l'intestin et les canaux biliaires; quoique Zakliarow (1919) mette en doute le bien-fondé de cette assertion, il est clair que la localisation de ces vers en dehors du système sanguin est tout au plus passagère sinon accidentelle.

(3) Une description plus exacte d'Unicacum rus:kowskii navait pas encore paru au moment de la rédaction du présent mémoire. 
Sous-famille Hapalolreminze Stunk., 1921.

Genre Hapalolrema Looss, 1899.

Espèce : H. constrictum (Leared, 1862).

- Genre Hapalorhynchus Stunk., 1922.

Espèce : H. gracilis Stunk., 1922.

Les formes appartenant aux Spirorchinæ, Spirorchis blandingi excepté, diffèrent si peu entre elles que la validité des espèces et même des genres est problématique. Ainsi le Spirorchis emydis Mc. Cal. est considéré par Stunkard (1923) comme étant identique Spirorchis innominata Ward et l'Henotosoma hæmatobium Stunk. est. suivant Mac Callum (1926), identique au Spirorchis chelydræ Mc Cal. Leurs dimensions ainsi que leur forme ne peuvent pas servir d'indication suffisante pour la définition d'une nouvelle espèce ou d'un genre nouveau, car ces vers changent facilement de forme et de grandeur par suite de l'élasticité de leur corps. Il en est de mème du nombre de testicules qui dépend parfois de la maturité et de l'àge du ver, comme Stunkard (1923, p. 199) l'a constaté. Quant à la grandeur des vers, elle peut dépendre aussi de leur âge. De nombreuses études comparatives se rapportant aux différentes périodes de la maturité permettraient seules d'établir une classification rationnelle. Abstraction faite de la valeur de la classification de la sous-fanmille des Spirorchinæ en espèces et en genres, il faut constater que le Spirorchis blandingi Mc Call. diffère nettement des autres représentants de cette sous-famille, et même davantage que les différents genres entre eux. Le Spirorchis blandingi forme le premier point du trait d'union entre les trématodes hématophages des tortues américaines et ceux des tortues européennes. Cette espèce représente la forme de passage entre les deux sous-familles.

Nous donnons ici la description du type européen, pour lequel nous proposons le nom de Spirhapalum polesianum n. g., n. sp.

\section{Description du ver adulte}

La forme du ver est plus ou moins ovale, métabolique en raison de la contractibilité du corps (fig. 1 et 2). Dimensions : $1 \mathrm{~mm}$., 18 à $2 \mathrm{~mm}$., 3 de long sur $0 \mathrm{~mm}$., 38 à $0 \mathrm{~mm}$., 65 de large.

Les contours sont hérissés de poils bacilliformes (fig. 3 ).

Le ver est pourvu de deux ventouses ovales: la buccale $(v b)$ longue de 69 à $76 \mu$ et large de 60 à $85 \mu$ et la ventrale $(v v)$ longue de 65 à $132 \mu$ et large de 74 à $123 \mu$. Cette dernière est située dans la partie antérieure du corps, vers le centre.

APPAREIL DigestiF. - Le pharynx fait défaut. L'œsophage s'étend 
en arrière en ligne sinueuse, formant einq ou six replis dont les dimensions progressent en même temps que le diamètre de l'organe lui-même augmente $(œ)$. Cet organe est entouré d'une enveloppe de
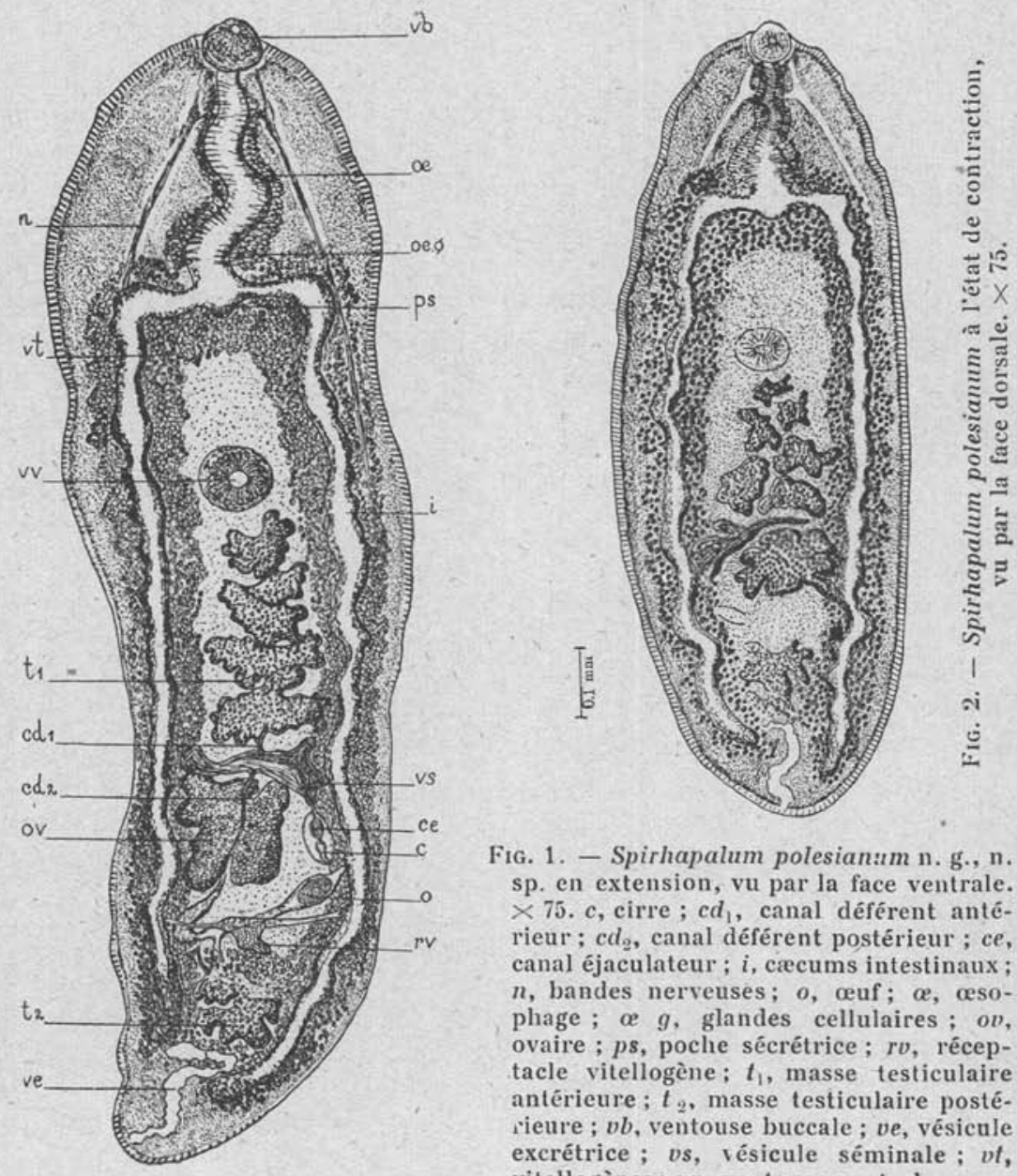

Fig. 1. - Spirhapalum polesianım n. g., n. sp. en extension, vu par la face ventrale. $\times 75$. $c$, cirre ; $c d_{1}$, canal déférent antérieur ; $c d_{2}$, canal déférent postérieur ; $c e$, canal éjaculateur ; $i$, crecums intestinaux ; $n$, bandes nerveuses; $o$, œuf; $\propto$, œsophage ; $\propto g$, glandes cellulaires ; $o v$, ovaire ; $p s$, poche sécrétrice ; $r v$, réceptacle vitellogène; $t_{1}$, masse testiculaire antérieure ; $t_{2}$, masse testiculaire postérieure; $v b$, ventouse buccale; ve, vésicule excrétrice; $v s$, vésicule séminale ; $v t$, vitellogènes; $v v$, ventouse ventrale.

glandes cellulaires allongées, débouchant par leur extrémité dans l'œsophage et s'accumulant à sa base, en donnant l'impression d'un renflement $(\propto g)$. A la fin du premier quart ou vers le cinquième du corps, l'œsophage se bifurque, en envoyant sur un court parcours à droite et à gauche les conduits intestinaux qui, en ce point, se recourbent subitement en arrière (i). A l'endroit de bifurcation du 
tube digestif se trouve une petite poche qui constitue la suite de l'œsophage ( $p s)$, et que je considère comme l'homologue de la vésicule sécrétrice des Sanguinicola (Ejsmont, 1925). Les cæcums intestinaux (i) parviennent presque jusqu'au bout du corps, formant une ligne irrégulière, remplie de la substance jaune, brune ou noire, preuve de la décomposition plus ou moins avancée des érythrocytes. La description ci-dessus se rapportant à la structure de l'appareil digestif s'accorde complètement avec celle de Stunkard (1923) pour le genre Spirorchis, où l'auteur décrit en même temps les relations histologiques.

Système Nerveux. - Le système nerveux correspond aussi à celui des Spirorchis. La commissure nerveuse, réunissant les deux ganglions, se trouve située au quart de la distance entre la ventouse
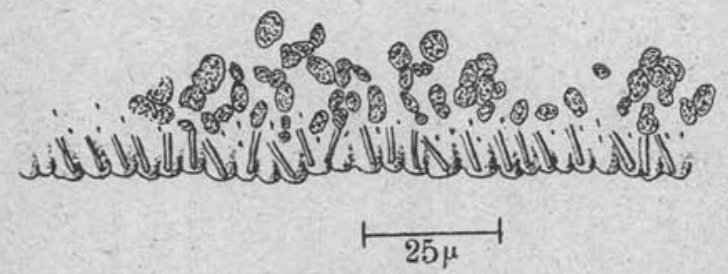

FIG. 3. - Fragment du bord du corps de Spirhapalum polesianum $\times 600$.

buccale et la bifurcation. Des ganglions cérébroïdes partent des bandes nerveuses $(\boldsymbol{n})$ vers l'extrémité antérieure et postérieure.

Appareil excréteur. - En ce qui concerne cet appareil, on n'aperȩoit que la vésicule excrétrice en forme de tuyau courbé (ve) qui n'atteint pas l'extrémité du corps, ce qui a lieu aussi chez les autres Spirorchidés.

ApPareil gÉnital. - Màle. - Les testicules sont accumulés en deux masses : l'une antérieure, l'autre postérieure $\left(l^{\prime}\right.$ et $\left.t^{2}\right)$ et elles sont séparées par l'ovaire $(o v)$ et les canaux génitaux. La masse antérieure occupe l'espace situé entre la ventouse ventrale et la vésicule séminale $(\nu s)$ et se compose ordinairement de six lobules testiculaires à contours irréguliers, placés en ligne brisée, dont les angles sont dirigés vers les deux bords de l'animal. La sinuosité de cette ligne dépend de l'allongement du ver. Souvent les testicules particuliers sont tellement rapprochés et serrés qu'ils semblent former une file ininterrompue ne laissant pas même distinguer les testicules particuliers qui, en effet, sont joints entre eux à l'aide de canaux efférents. Ces canaux efférents, précisément au moment où ils sont remplis de sperme, peuvent s'élargir considérablement, effaçant les limites entre les testicules. Les testicules grandissent 
successivement dans la direction postérieure. La masse testiculaire postérieure $\left(t^{2}\right)$ se compose d'un ou de deux testicules, intimement liés entre eux. Les deux masses, à l'exemple des Hapalotrema, se joignent dans la vaste vésicule séminale (vs) à l'aide de canaux déférents très fins. Le canal déférent postérieur $\left(c d^{2}\right)$ ést évidemment beaucoup plus long et, parcourant en ligne médiane irrégulière au-dessus des conduits génitaux femelles et de l'ovaire, se dirige vers la partie moyenne de la vésicule séminale. Le canal déférent antérieur $\left(c d^{\prime}\right)$ part du centre du lobule postérieur de la masse testiculaire antérieure et se dirige également vers la partie centrale de la vésicule séminale, vis-à-vis du canal déférent postérieur. La vésicule séminale a une forme irrégulière et se trouve d'habitude horizontalement et un peu de travers, allant du côté droit au còté gauchè ; elle sépare la masse testiculaire antérieure de l'ovaire. Cependant, dans une des préparations, elle est plutôt verticale, se rapprochant ainsí de sa situation chez les Spirorchis. La vésicule séminale est remplie de sperme qui, en la distendant, lui donne un aspect irrégulier. L'extrémité antérieure, c'est-à-dire droite, de la vésicule n'a pas d'issue ; l'extrémité postérieure, c'est-àdire gauche, forme le plus souvent deux cæcums et un troisième petit conduit étroit, dirigé en arrière, entre presque aussitôt dans une poche du cirre (fig. $4, p c$ ) ; c'est le canal éjaculateur (ce). La vésicule séminale du ver que nous décrivons et celle des Hapalotrema sont homologues, ayant la forme que l'on observe chez les Spirorchinæ. Mais puisque cette vésicule n'est pas différenciée en canal déférent et en vésicule séminale, il est par conséquent plus juste de considérer cet organe, eu égard à sa valeur fonctionnelle, comme un canal déférent, remplissant en mème temps la fonction de vésicule séminale. Les auteurs américains (Ward, Stunkard, Mac Callum), en décrivant les formes appartenant aux Spirorchi$n æ$, considèrent cet organe, sortant des testicules, comme une vésicule séminale et nient l'existence du canal déférent. Quoiqu'une telle manière de voir ne change pas l'état des choses, au point de vue de la nécessité fonctionnelle, il faut considérer le canal déférent comme un organe primitif et la vésicule séminale comme une différenciation consécutive d'une certaine partie du canal déférent avec lequel elle peut quelquefois se fusionner. En réalité, la vésicule séminale sans canal déférent perd sa raison d'être, tandis que ce dernier peut accomplir en même temps les deux fonctions, ce qui a lieu par exemple chez les Sanguinicola. De même que chez les Hapalotrema, une assez vaste poche du cirre entoure le canal éjaculateur $(c e)$ et le cirre renflé $(c)$. Ces deux parties peuvent être séparées par un rétrécissement marqué ; alors le canal éjacula- 
teur apparaît sous la forme d'un organe ovale rempli de spermatozoïdes. La poche du cirre semble être privée des cellules de la glande. prostatique de même que chez la plupart des Spirorchidés. L'orifice mâle se trouve au voisinage de l'orifice femelle, sur la face ventrale, à proximité de la branche gauche de l'intestin et souvent sous celle-ci.

Femelle. - L'appareil génital femelle est simple. L'ovaire est lobé et se trouve du côté droit, au delà de la masse testiculaire antérieure, dont il est ordinairement séparé par la vésicule séminale.

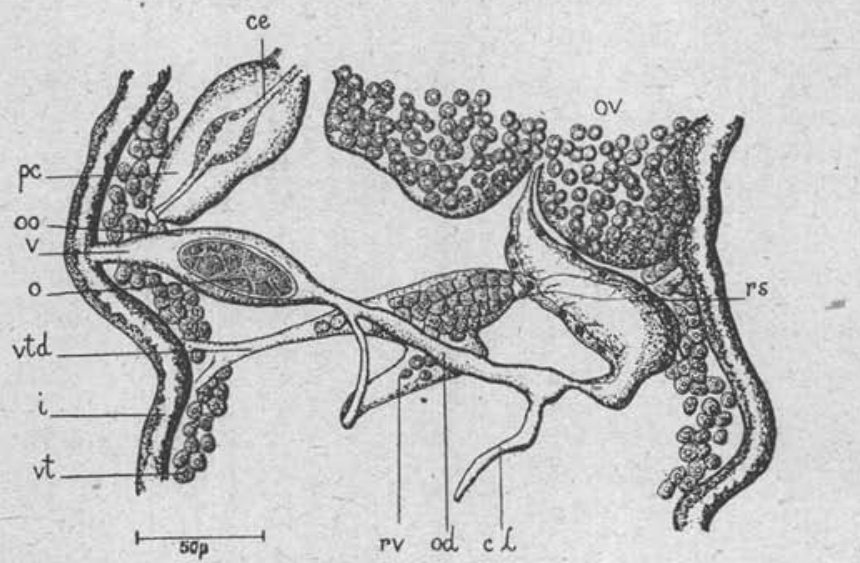

Fig. 4. - Canaux génitaux femelles de Spirhopalum polesianum $\times 270 . c L$, canal de Laurer ; od, ootype; $p c$, poche de cirre ; rs, réceptacle séminal ; $v$, vagin (metraterme); vtd, vitelloducte. Les autres abréviations sont les mêmes que pour la fig. 1 .

L'ovaire est beaucoup plus grand que les testicules particuliers. L'oviducte (fig. $4, o d$ ) prend naissance au milieu du bord postérieur de l'ovaire et, se dirigeant dorsalement en arrière et à droite, forme un renflement où s'accumulent les spermatozoïdes $(r s)$.

Ward (1921) et Stunkard (1923) appellent ce renflement receptaculum seminis uterinum, mais, puisqu'au point de vue topographique, cette vésicule n'est pas en rapport avec l'utérus, mais avec l'oviduete, cette dernière expression ne peut être considérée comme exacte (Ejsmont, 1925, p. 952). Nous avons souvent rencontré dans cette partie un grand nombre de spermatozoïdes mobiles. De ce point l'oviducte tourne à gauche et légèrement en avant en donnant bientôt l'étroit canal de Laurer $(c L)$ dirigé obliquement en arrière. L'oviducte, continuant à cheminer dans la direction qu'il avait en sortant du réceptacle séminal, court vers le côté gauche de 
l'animal, s'adjoignant, en avant de l'ootype, le vitelloducte. L'utérus n'est représenté que par l'ootype ovale $(o o)$ que l'on voit distinctement, même lorsqu'il ne contient pas d'œuf. Je n'ai pas constaté d'éléments glandulaires appartenant au corps de Mehlis. L'ootype se prolonge en vagin ou metraterme $(v)$, s'ouvrant sous le cæcum intestinal gauche, non loín de l'orifice màle. Les glandes vitellogènes $(v t)$ se composent de nombreux éléments occupant l'espace compris entre le point de bifurcation de l'intestin et l'extrémité du corps, aussi bien à l'extérieur qu'à l'intérieur des cæcums. Les glandes vitellogènes des deux côtés se rejoignent dans la région des testicules postérieurs, les entourant de toute part. Le vitelloducte transversal $(v t d)$, caractéristique dans toute la famille, part des deux glandes vitellogènes ventralement, au-dessous des orifices génitaux et de l'ovaire et il forme dans sa partie centrale une vésicule vitellogène $(r v)$ qui envoie dorsalement vers l'extrémité antérieure du corps un canal débouchant du côté dorsal dans l'oviducte en avant de l'ootype.

\section{Euf et miracidium}

Euf. - On peut rencontrer souvent dans l'ootype un jeune œuf de forme ovale (o) mesurant $54 \mu \times 30 \mu$, qui est éliminé promptement par les canaux génitaux dans la circulation du sang de l'hôte ; il y grandit et y mûrit. Les œufs sont emportés avec le courant sanguin par les voies circulatoires et s'enfoncent dans les divers tissus, surtout dans les poumons, les reins, la rate et le foie. Dans le mésentère des intestins, contrairement à l'assertion d'autres auteurs, je n'ai constaté aucune infection. Stunkard (1923) prétend que les œufs des Spirorchidés, pour passer à l'extérieur, doivent se frayer un chemin d'une manière passive à travers les tissus, dans la direction de la lumière du tube digestif. Cependant une telle opération ne pourrait avoir de succès, parceque nous savons d'autre part (Monticelli, 1896 ; Loos, 1902 ; Ward, 1921 ; Mac Callum, 1926), que, de même que chez les Sanguinicola (Scheuring, 1922 ; Ejsmont, 1925), les æufs, ayant traversé des vaisseaux sanguins pour passer dans des tissus serrés, s'y enkystent. Les branchies des poissons, qui se trouvent en contact direct avec le milieu extérieur, contituent la voie la plus favorable pour les oufs des A porocotylidæ ; au contraire, chez tous les hôtes respirant à l'aide de poumons, la condition essentielle pour les œufs de trématodes est de se frayer un passage par le tube digestif ou par les urètres. A mon avis, les œufs des Spirorchidæ parviennent aú tube digestif, des poumons par la trachée, ou du foie par le canal cholédoque, et aux 
urètres, en venant directement des reins. Cette manière de voir s'accorde aussi avec le fait, que ces organes sont précisément les plus abondamment. infectés; en outre, les poumons des tortues semblent constituer pour les œufs des Spirorchidæ un endroit de choix, comme les branchies des poissons pour les œufs des A porocotylida, ce qui est confirmé, non seulement par mes propres observations, mais aussi par celles des autres auteurs (1). Le chemin, que doivent parcourir les embryons ou les oufs, exerce aussi une influence sur le lieu de l'éclosion du miracidium, c'est-à-dire qu'elle se produit soit dans l'organisme même de l'hôte (A porocotylidx), soit en dehors de celui-ci (Spirorchidæ, Schistosomidx) (2).

Dans toutes les tortues disséquées, j'ai rencontré des œufs en grand nombre. Chez les tortues examinées jusqu'au 22 mai, on trouvait exclusivement des œufs jeunes, formés de cellules vitellogènes vacuolisées et d'une sphère blasthomérique de grandeur moyenne ; mais le 30 mai les poumons de la tortue, outre des œufs jeunes, renfermaient aussi des oufs contenant des miracidiums mobiles. Toutes les tortues examinées ultérieurement jusqu'au moment de l'interruption de mes recherches (3 juillet), présentaient beaucoup d'œufs à différents stades de développement. Il faut voir ici une adaptation à la saison, ce que j'ai décrit également à propos des Sanguinicola. Pendant la saison la plus fraîche, on rencontre de moins en moins d'œufs contenant des miracidiums, parceque les uns libèrent à temps leur miracidium, tandis que les autres, après avoir atteint le développement complet et n'ayant pu se libérer de l'embryon, disparaissent. La saison fraîche retarde le développement des œufs jeunes; aussi au printemps ne rencontronsnous que des œufs jeunes. A l'approche de la saison plus chaude, les jeunes œufs se développent vite, ce qui facilite l'observation de ceux-ci dans les différentes phases de leur formation.

Ls œufs, après leur élimination de l'organisme du ver, semblent changer le rapport réciproque du grand et du petit axe au profit de ce dernier, ce que l'on peut attribuer à la vacuolisation importante des cellules vitellogènes, qui distendent la mince coquille. Leur forme ressemble à celle des œufs des Spirorchis, mais ils sont, quel que soit leur âge, transparents, à coque fine et semblent être dépourvus d'opercule. En moyenne, leur grandeur est plus considérable que celle des œufs des espèces particulières à la sous-famille des Spirorchinx, quoiqu'on rencontre dans ce groupe des formes

(1) Par exemple Mac Callum (1926) dit: « ....Les œufs sont principalement répartis dans le tissu pulmonaire de tous les animaux infectés $n$ (p. 97).

(2) Comparer Ejsmont (1925), p. 925. 
surpassant de beaucoup notre ver (Henotosoma hamatobium). Puisque les œufs, flottant dans le sang, grandissent continuellement jusqu'à la formation des miracidiums, on en trouve de toutes dimensions ; le plus petit œuf, observé par moi hors de l'organisme maternel, mesurait $76 \mu \times 53 \mu$. Les oufs qui se trouvaient dans la rate de l'hôte avaient en géníral les dimensions les plus inférieures. Les œufs renfermant un embryon mobile complètement développé ont les proportions les plus stables. Je donne ici les dimensions d'une série d'œufs, exprimées en microns :

$\begin{array}{llll}100 \times 68 & 140 \times 80 & 148 \times 110 & 160 \times 88 \\ 122 \times 74 & 140 \times 92 & 156 \times 88 & 170 \times 110 \\ 126 \times 82 & 146 \times 84 & 158 \times 90 & 176 \times 90\end{array}$

Il est possible que la finesse de la coque des oufs de cette espèce puisse expliquer l'absence d'opercule.

Alors que dans l'ootype l'œuf est complètement rempli de cellules vitellogènes à structure finement granulée $(o)$, dans le sang au contraire, quoiqu'on y rencontre des œufs se trouvant, encore, dans la période précoce de formation de l'embryon, l'œuf présente déja des cellules vitellogènes relâchées, plus grandes et à l'aspect alvéolaire (1) (fig. 5, a). Le liquide de ces cellules dónne une réaction glycogénique intense. Parfois dans cette substance liquide on aperçoit des granules de glycogène ayant des mouvements browniens. Dans la masse embryonnaire non différenciée au début. apparaissent d'abord les ébauches des ocelles, qui occupent une position symétrique dans la partie latérale de l'œuf (fig. $5, b$ ). A mesure que progresse l'agglomération du pigment, les deux ébauches se rapprochent.

Dans la phase avancée, l'embryon en état de formation repousse les cellules vitellogènes jusqu'à la périphérie, et, au moment de son complet développement, il ne reste que deux cellules, éloignées aux deux pôles opposés du grand axe (fig. $5, c)$. Les cils, comme on devait s'y attendre, ne se forment qu'à la fin.

En raison de la transparence de la coque, on voit bien la structure du miracidium pendant son séjour dans l'œuf. Les dimensions des miracidiums complètement développés, sont exprimées en microns :

$\begin{array}{rr}96 \times 50 & 122 \times 66 \\ 118 \times 68 & 126 \times 54 \\ 120 \times 64 & 130 \times 74\end{array}$

(1) Bien que les cellules vitellogènes grandissent, leur union néanmoins devient plus lâche qu'auparavant, ce qui s'explique par la diminution du nombre des cellules et l'accroissement prompt des dimensions de l'œuf lui-mẽme. 
Miracidium. -- La forme fondamentale du miracidium est ovale avec la partie antérieure conique. Tout le corps, à l'exception de l'extrémité antérieure, est couvert de cils de la même longueur, mesurant environ $12 \mu$. L'extrémité antérieure, en forme de trompe, contient l'orifice buccal, conduisant dans le sac digestif en massue

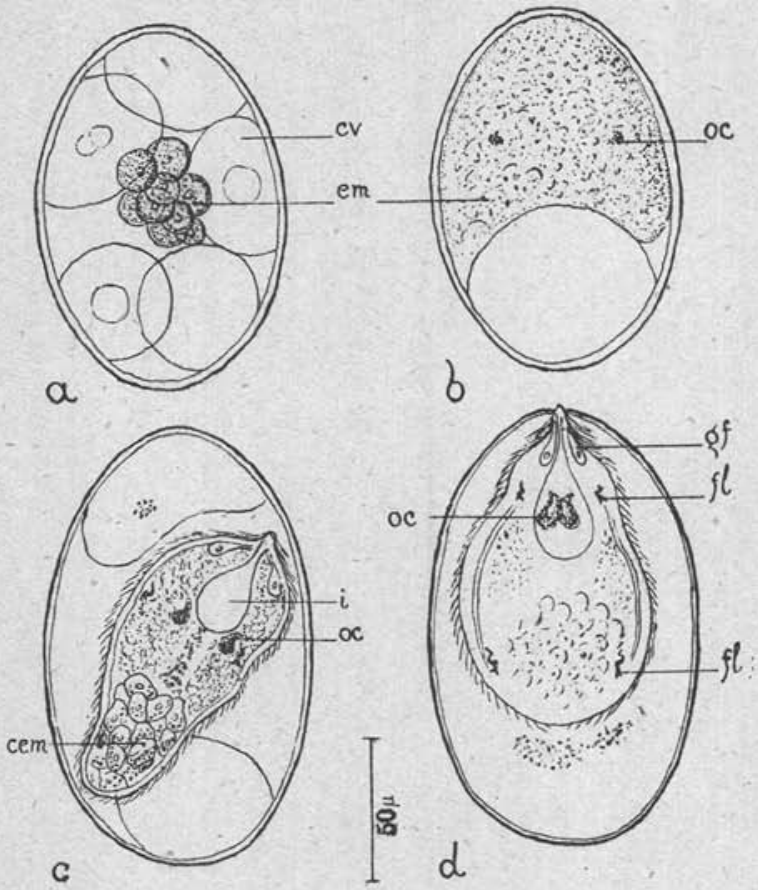

Fig. 5. - Cufs de Spirhapalum polesianum dans les différentes phases du développement de l'embryon. $a$, œuf fraichement expulsé de l'ootype; $b$, œuf contenant l'embryon au commencement du développement des ocelles; $c$, œuf avec un miracidium formé ; $d$, œuf avec le miracidium au moment de l'éclosion. Toutes les figures $\times 300$. cem, cellules germes; $c v$, cellules vitellogènes; $\mathrm{em}$, embryon ; $f$, flammes vibreatiles; $g f$, glandes céphaliques; $i$, sac digestif : oc, ocelles.

(fig. 5, i). Des deux côtés de ce dernier, à proximité des parois du corps, sont situées les glandes céphaliques frontales ( $g f$ ), beaucoup plus petites que celles qui caractérisent les larves des autres Spirorčhidés et des Schistosomidés. Les taches de pigment $(o c)$ ressemblent par leurs caractères et par leur topographie aux taches décrites par Stunkard (1923) dans le genre Spirorchis. Elles peuvent prendre entre elles des positions diverses suivant les mouvements du miracidium. A l'extrémité postérieure du corps se trouve une 
grande accumulation des cellules germinales (cem). Je n'ai pu constater, dans l'appareil excréteur, que les flammes vibratiles ( $f l$ ). La paire antérieure se trouve entre les ocelles et les parois latérales du corps ; la paire postérieure est placée au niveau du dernier tiers de l'amas des cellules embryonnaires, à la même distance des parois latérales que la paire antérieure. Les cils vibratiles sont dirigés en avant. Les deux flammes, de chaque côté, semblent être réunies par un vaisseau oblong (1).

Comme je l'ai dit plus haut, l'embryon en mouvement se trouve le plus souvent sifué entre deux cellules vitellogènes, se plaçant, soit dans la direction de l'axe longitudinal de l'œuf, soit transversalement, ou bien obliquement. Outre les mouvements des muscles et des cils, le miracidium exécute des mouvements intenses, qui sont en rapport avec le changement de sa position ; par exemple, il tourne autour de son grand axe, ou il remonte le long de la coque de l'œuf, repoussant devant lui la boule vitellogène ; souvent il frappe la coque avec son extrémité antérieure. L'absence presque complète d'éléments vitellogènes et les efforts très énergiques faits par le miracidium pour sortir de l'œuf, témoignent que l'éclosion approche. Quoique je n'aie pas vu le mouvement de l'éclosion de l'embryon, j'ai pu cependant observer une fois un miracidium sur le point de quitter la coque de l'œuf : il frappait de temps en temps avec son extrémité conique antérieure, un des pôles de l'œuf, ce qui occasionna une petite brèche, mais je n'ai pas réussi à observer sa sortie complète.

\section{Position systématique dans la famille des Spirorchidæ}

Comme il résulte de la précédente description, notre ver possède des caractères propres à chacune des sous-familles de la famille des Spirorchidæ, par conséquent, il constitue une forme de passage.

Les deux sous-familles diffèrent entre elles, d'après Stunkard, par l'existence, soit d'une faible ventouse buccale, soit de deux ventouses, ensuite par la topographie de l'ovaire relativement à l'axe longitudinal du corps et aux testicules, puis par le développement de la poche du cirre, par la situation des orifices génitaux

(1) Mathias (1925) en raison de la ressemblance entre la structure deś miracidiums de Spirorchis et d'Hapalotrema fait observer que le miracidium de ce dernier, pour lequel Monticelli ne dessine qu'une seule paire de flammes, devait avoir aussi deux paires de flammes vibratiles. Je suis enclin à admettre son opinion, mais plutôt par égard à la classification génétique des formes adultes. Voir mon tableau comparatif (1925, p. 949 et p. 922). 
par rapport à la surface du corps (1) et à son axe longitudinal, ainsi que par le caractère de l'œuf. Choisissons les traits communs à notre ver et aux genres typiques de deux sous-familles, c'est-à-dire aux genres Spirorchis et Hapalotrema.

Traits communs avec les Spirorchis. - La forme de l'œsophage, la topographie de l'ovaire, des conduits et des orifices génitaux vis-à-vis de l'axe longitudinal du corps, le rapport de l'ootype au vagin (métraterme), la forme de l'œuf, le miracidium avec les taches ophtalmiques.

Traits communs avec les Hapalotrema. - Deux ventouses, la présence des soies, deux masses testiculaires et avec cela deux canaux déférents, la présence de la vésicule séminale et d'une vaste poche du cirre.

Il s'ensuit donc que la division de la famille Spirorchida en deux sous-familles perd sa raison d'être. Je propose pour le nouveau ver le nom générique de Spirhapalum, qui rappelle l'étymologie des noms des deux sous-familles.

Quant au Spirorchis blandingi Me Call., il faut souligner que cette forme, possédant tous les caractères propres aux Spirorchis et aux geñres voisins, en diffère pourtant par un trait très important et essentiel, autorisant l'élimination de l'ancien genre ; c'est la possession de.deux masses testiculaires. L'auteur lui-même, qui soutient que la position des testicules " en avant de l'ovaire " (1926, p. 98) est un des traits caractérisant le genre Spirorchis, n'est pas conséquent avec lui-même en conservant pour cette forme l'ancienne dénomination générique. Je propose de séparer cette

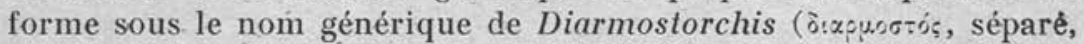
divisé). On observe en effet chez elle la division d'une masse primitive des testicules en deux, séparées par l'ovaire et les voies génitales. C'est un caractère propre aux Hapalotremina, mais cette forme conserve encore tous les autres caractères des Spirorchinæ. Ce genre forme la première étape du rapprochement des conduits génitaux et des orifices vers la partie antérieure du corps.

Si nous comparons le groupe entier des trématodes hématophages, nous voyons, qu'avec le passage des voies $e v$ des orifices génitaux dans la partie antérieure du corps, la maśse des testicules primitivement unique (Aporocotylidæ et Spirorchinæ) (fig. 6, a) commence à se diviser et que par les genres Diarmostorchis et Spirhapalum, chez lesquels un ou deux testicules se séparent de

(1) Stunkard, acceptant la position dorsale des orifices génitaux comme caractéristique de la sous-famille des Hapalolremine, soutient que l'Hapalotrema constrictum possède aussi ces rapports, mais il ne le démontre pas; Monticelli admet, dans sa description et dans ses dessins, la position ventro-latérale. 
l'ensemble (fig. $6, b$ ), les testicules forment déjà une grande masse postérieure et une plus petite antérieure (Hapalotrema) (fig. 6, c). Pendant cette migration, l'ovaire est repoussé aussi continuelle-

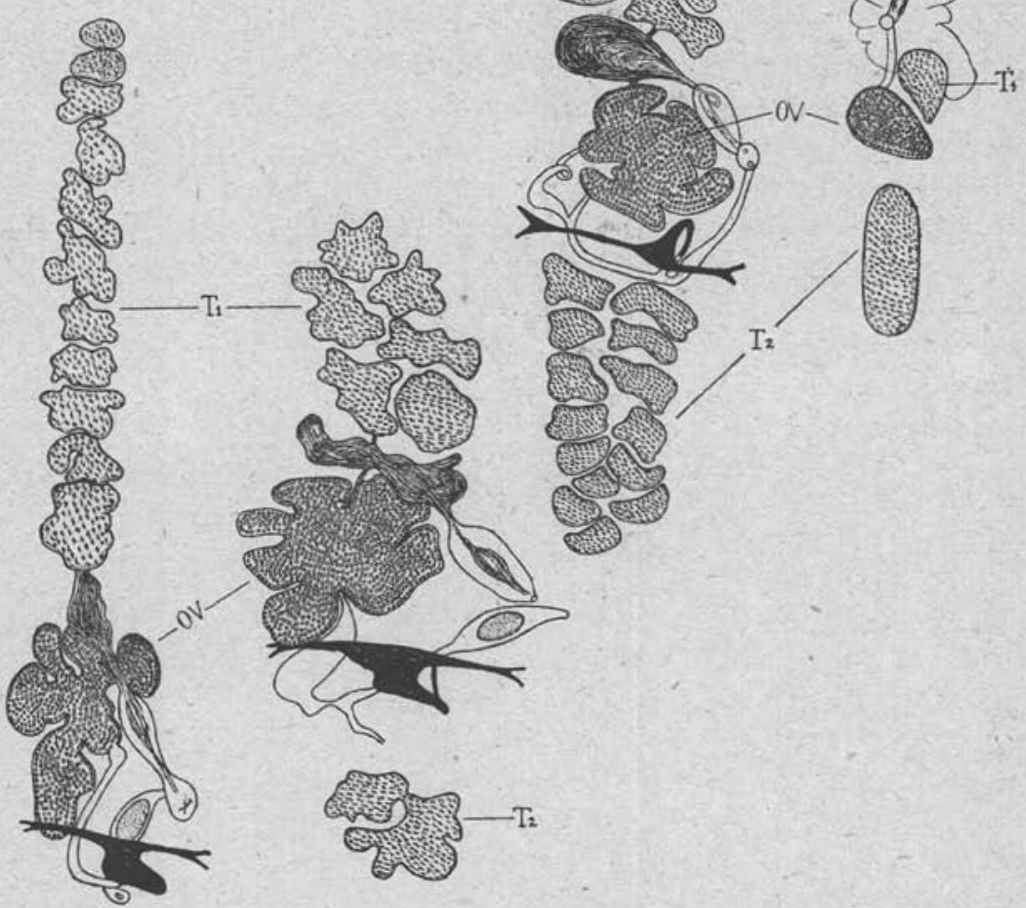

Fıg. 6. - Tableau comparatif de la disposition des organes génitaux dans la famille de Spirorchida. a, Spirorchis Mc. Call et les genres voisins; $b$, Spirhapalum n. g. ; c, Hapalotrema Loos ; d, Hapalorhynchus Stunk; $a$, selon Ward, 1921 ; c, suivant les figures de Monticelli, 1896 et de Loos, $1902 ; d$, selon Stunkard, 1922-1923; ov, ovaire; $T_{1}$, masse testiculaire antérieure; $T_{2}$, masse testiculaire posterieure.

ment en avant par les conduits femelles. En continuant leur chemin les orifices génitaux laissent en arrière l'ovaire et quittent complètement le champ testiculaire (Hapalorhynchus) (fig. 6, d). Chez les Schistosomidr nous voyons que cet acheminement est poussé plus loin encore (1).

(1) Voir mon tableau comparatif et le texte respectif de 1925. 
En ce qui concerne les deux masses testiculaires des Hapalorhynchus, qui ne se composent que de deux testicules séparés par l'ovaire, il faut accepter l'explication suivante : les conduits génitaux femelles se sont retirés de l'endroit précédent sous l'ovaire, et les orifices génitaux avec les conduits mâles ont quitté la région testiculaire au moment où l'ovaire s'approche du dernier testicule antérieur, constituant ainsi la masse antérieure. Il ne reste de la masse postérieure que le testicule plus grand, formant peut-être quelques testicules fusionnés; le reste disparait.

Nous voyons done que la séparation qui existait jusqu'ici entre les Spirorchinæ et les Hapalotreminæ disparait. En envisageant la corrélation existant entre le degré d'évolution de l'hòte et le trématode correspondant, qui vit dans son sang, on peut considérer les parasites qui seront peut-être découverts dans le sang des amphibiens, comme devant combler, au moins en partie, la lacune qui subsiste encore entre les Aporocotylidæ et les Spirorchidæ, si toutefois ces formes n'ont pas disparu avee les amphibiens fossiles stégocéphales ou les reptiles cotylosauriques. Par contre, les formes, qui constituaient la transition entre les Spirorchida et les Schistosomidæ pourraient se rencontrer dans le sang des crocodiles et des squamates (plagiotremes), à moins qu'elles n'aient disparu avec les reptiles ptérosauriens.

\section{RÉSUMÉ}

1. Nous avons découvert dans le sang du cœur d'Emys orbicularis L. de Polésie, en Pologne, des individus mûrs, et dans la rate des exemplaires jeunes, d'un nouveau représentant de la famille des Spirorchidæ Stunk., Spirhapalum polesianum n. g., n. sp.

2. Les-divers tissus de l'hôte contenaient les oeufs de ce ver et permirent la constatation de leur développement saisonnier.

3. Spirhapalum polesianum forme par la conformation de ses organes la transition entre les sous-familles des Spirorchinæ Stunk. et des Hapalotreminæe Stunk., abolissant les différences existant entre ces deux sous-familles.

4. La découverte de cette nouvelle forme permet d'élucider le problème philogénétique des trématodes hématophages, éclairant le déplacement orthogénétique des organes et des orifices génitaux pendant l'évolution génétique.

5. Spirorchis blandingi Mac Call. constitue le premier échelon du passage entre les deux sous-familles des Spirorchidæ; je le considère comme devant former le type d'un genre nouveau, le genre Diarmostorchis. 
6. Les formes intermédiaires, parmi les trois familles de trématodes hématophages, pourraient habiter les vertébrés à un degré d’évolution correspondant.

\section{BiblogRaphIE}

Essmont (L.). - Morphologische, systematische und entwickelungs geschichtliclie Untersuchungen an Arten des Genus Sanguinicola Pleln. Bull. de l'acad. polon. d. sciences el d. leltres. Cl. d. sc. math. el natur., Série B., 1926, p. 877-966.

Leanev(A.). - Description of new parasite found in the heart of the edible Turtle. Quart. journ. of micr. Sc., II, 1862, p. 168-170.

Looss '(A.). - Weitere Beiträge zur Kenntnis der Trematoden-Fauna Aegyptens zugleich Versuch einer natürlichen Gliederung des Genus Distomum Retzius. Zool. Jahrb. Syst., XII, 1899, p. 521-784.

- Ueber neue und bekannte Trematoden aus Seeschildkröten, Nebst Erörterungen zur Systematik und Nomenclatur. Zool. Jahrb., Sysl., XVI, 1902, p. 411-894.

Mac Callum (G.-A.). - Notes on the Genus Telorchis and other Trematodes. Zoopathologica (N. Y. zool. soc.), I, 1918, p. 81-98.

- Studies in Helminthology. Zoopathologica, 1, 1921, p. 138-284

- Revue du genre Spirorchis Mac Callum. Ann. de Parasilologie, IV, 1926, p. $97-103$.

Mathias (P.). - Recherches expérimentales sur le cycle évolutif de quelques trématodes. Bull. biolog. de la Fran. et de la Belg., LIX, 1925, p. 1-124.

MoNTiceld (F.-S.). - Di un ematozoo della Thalassochelys caretla Linn. Intern. Monatsch. f. Anal. u. Physiol., XIIl, 1896, p. 141-172.

Odhner (T.). - Aporocolyle simplex n. g. n. sp., ein neuer Typus von ektoparasitischen Trematoden. Centr. f. Bakl. Paras. u. Inf., XXVII, 1900, p.62-66.

- Zum natürlichen System der digenen Trematoden. V. Die Phylogenie des Bilharzia-Typus. Zool. Anz., XLI, 1912, p. 5t-71.

Scheurivg (L.). -- Der Lebenszyklus Sanguinicola inermis Plehn. Zool. Jahrb., Ont. u. Entw., XXXVI, 1922.

Stunkaro (H.-W.). - Notes on North American Blood Flukes. American Museum novitates, $\mathrm{n}^{\circ} 12,1921, \mathrm{p} .1-5$.

- Two new Genera of North American Blood Flukes. Amer. Mus. novit, $n^{\circ} 39,1922$, p. 1-8.

Studies on North American Blood Flukes. Bull. of the americ. Mus, of nat. hist., XLVIII, 1923, p. 165-221.

A new blood fluke, Uniçecum ruszkowskii ı. g. n. sp.; a contribution to the relationship of the blood infesting trematodes. Jontn. of Paras., XII, 1926, p. 164-165.

Ward (H.-B.). - A new Blood Fluke from Turtles. Journ. of Paras., VII, 1921, p. 114-129.

ZakHarov (N.). - Kobnarujeniiu Bilharziella polonica u donskih ptiz. Trudy Obsch. veterin. vratsch. Vsevel. Iojsk, Donsk. Novotscherkask, wyp. I, 1919, p. 1-14 (en russe).

Laboraloire de Zoologie el de Parasilologie de l'Ecole vétérinaire de Varsovie. 